\title{
Thin magnesium layer confirmed as an antibacterial and biocompatible implant coating in a co-culture model
}

\author{
SARAH ZAATREH ${ }^{1}$, DAVID HAFFNER ${ }^{2}$, MADLEN STRAUSS $^{1}$, THOMAS DAUBEN $^{1}$, CHRISTIANE ZAMPONI $^{2}$, \\ WOLFRAM MITTELMEIER $^{1}$, ECKHARD QUANDT $^{2}$, BERND KREIKEMEYER $^{3}$ and RAINER BADER ${ }^{1}$ \\ ${ }^{1}$ Biomechanics and Implant Technology Research Laboratory, Department of Orthopaedics, University Medicine Rostock, \\ D-18057 Rostock; ${ }^{2}$ Institute for Materials Science, Faculty of Engineering, University of Kiel, D-24143 Kiel; \\ ${ }^{3}$ Institute of Medical Microbiology, Virology and Hygiene, University Medicine Rostock, D-18057 Rostock, Germany
}

Received August 30, 2016; Accepted November 21, 2016

DOI: $10.3892 / \mathrm{mmr} .2017 .6218$

\begin{abstract}
Implant-associated infections commonly result from biofilm-forming bacteria and present severe complications in total joint arthroplasty. Therefore, there is a requirement for the development of biocompatible implant surfaces that prevent bacterial biofilm formation. The present study coated titanium samples with a thin, rapidly corroding layer of magnesium, which were subsequently investigated with respect to their antibacterial and cytotoxic surface properties using a Staphylococcus epidermidis (S. epidermidis) and human osteoblast (hOB) co-culture model. Primary hOBs and $S$. epidermidis were co-cultured on cylindrical titanium samples (Ti6Al4V) coated with pure magnesium via magnetron sputtering (5 $\mu \mathrm{m}$ thickness) for 7 days. Uncoated titanium test samples served as controls. Vital hOBs were identified by trypan blue staining at days 2 and 7 . Planktonic $S$. epidermidis were quantified by counting the number of colony forming units (CFU). The quantification of biofilm-bound $S$. epidermidis on the surfaces of test samples was performed by ultrasonic treatment and CFU counting at days 2 and 7. The number of planktonic and biofilm-bound S. epidermidis on the magnesium-coated samples decreased by four orders of magnitude when compared with the titanium control following 7 days of co-culture. The number of vital hOBs on the magnesium-coated samples was observed to increase $(40,000$ cells $/ \mathrm{ml})$ when compared with the controls $(20,000$ cells $/ \mathrm{ml})$. The results of the present study indicate that rapidly corroding magnesium-coated titanium may be a viable coating material that possesses antibacterial and biocompatible
\end{abstract}

Correspondence to: Ms. Sarah Zaatreh, Biomechanics and Implant Technology Research Laboratory, Department of Orthopaedics, University Medicine Rostock, 142 Doberaner Street, D-18057 Rostock, Germany

E-mail: sarah.zaatreh@med.uni-rostock.de

Key words: implant-associated infections, fast corrosion surface, co-culture, magnesium coating, titanium alloy, Staphylococcus epidermidis, human osteoblasts properties. A co-culture test is more rigorous than a monoculture study, as it accounts for confounding effects and assesses additional interactions that are more representative of in vivo situations. These results provide a foundation for the future testing of this type of surface in animals.

\section{Introduction}

A total of 375,000 hip and knee endoprostheses implants are performed in Germany each year (1). In $1.5 \%$ of cases, implant-associated infections (IAI) occur following total joint arthroplasty (2). Despite the relatively low prevalence of IAI, it may have severe consequences for patients, physicians and the health care system (3). In the majority of cases, infections are initiated by Staphylococcus aureus, a biofilm-forming coagulase-negative Staphylococcus epidermidis (S. epidermidis), or gram-negative species (4). Early infections occur within weeks following surgery and are usually acquired intraoperatively. In addition, IAI may manifest as a late infection, occurring between 3 and 24 months following surgery, resulting from hematogenous spreading of the bacteria from other foci $(5,6)$. IAI requires local and systemic antimicrobial therapy, which necessitates the removal of the contaminated endoprosthetic implant and insertion of a temporary cement implant (spacer) loaded with antibiotics (7-9).

There is currently a requirement for novel endoprosthetic materials with optimal surface and mechanical characteristics that prevent bacterial biofilm formation avoid bacterial resistance mechanisms and mediate biocompatibility and osseointegration (10). In recent years, magnesium has emerged as a promising implant material solution. Magnesium has various favourable properties, as it is a freely accessible metal and an essential cation serves an important role in the metabolism of human cells $(11,12)$. The biocompatibility of magnesium as a coating is influenced by the corrosion rate, corrosion products and the varying $\mathrm{pH}$-value of the solution (13). A previous in vitro study revealed that rapidly corroding magnesium in a thin layer on titanium (Ti6Al4V) was a suitable candidate for implant coatings with antimicrobial and biocompatible properties (14). Monocultures of S. epidermidis and human osteoblasts (hOB) revealed that magnesium coatings may prevent initial bacterial adhesion while remaining 
biocompatible according to the cytotoxicity test standards of the International Organisation for Standardisation (15). In contrast to monocultures, co-cultures provide a more comprehensive model of the development of the infection, as demonstrated in a previous study (16).

The aim of the present study was to investigate the antibacterial potential and cytotoxic effects of a thin, rapidly corroding magnesium coating on titanium samples in a co-culture model of S. epidermidis and hOBs.

\section{Materials and methods}

Titanium samples coated with pure magnesium. The present study used Ti6Al4V discs (DOT GmbH, Rostock, Germany) coated with pure magnesium for the experiments. Uncoated Ti6Al4V discs (a common implant material) served as a control. The discs were $11 \mathrm{~mm}$ in diameter and $2.5 \mathrm{~mm}$ in thickness. Magnesium-sputtering targets (diameter, $20 \mathrm{~cm} ; 3.5 \mathrm{~mm}$ thickness; purity, 3N5) were produced by Fhr Anlagenbau GmbH (Ottendorf-Okrilla, Germany). The magnetron sputtering process was conducted with a Von Ardenne CS730 cluster machine (Von Ardenne GmbH, Dresden, Germany) with a $200 \mathrm{~W}$ sputtering power and $2.3 \times 10^{3}$ mbar pressure. The samples were coated with layers of magnesium $(5-\mu \mathrm{m}$ thick) by breaking the vacuum and turning the samples. The temperature of the substrate holder did not surpass $76^{\circ} \mathrm{C}$, and all process parameters were performed in accordance with those described by Schlüter et al (17). The roughness $\left(R_{z}\right)$ of the surfaces was measured via 3D laser scanning microscopy (VK-X260, Keyence Corporation, Osaka, Japan). The Ti6A14V disc roughness was between 8 and $12.7 \mu \mathrm{m}$ for the magnesium-coated discs as determined in our previous study (14). The samples were Y-sterilized at $25 \mathrm{kGy}$ following the sputtering process.

Magnesium ion release and $\mathrm{pH}$ of the supernatants. The magnesium-coated Ti6A14V samples were pre-incubated for $24 \mathrm{~h}$ in Modified Eagle's osteogenic cell culture medium (MEM; EMD Millipore, Billerica, MA, USA) without calcium or antibiotics, containing $10 \%$ foetal calf serum (EMD Millipore), 1\% HEPES buffer (Gibco; Thermo Fisher Scientific, Inc., Waltham, MA, USA) and the following osteogenic additives: $100 \mathrm{nM}$ dexamethasone, $50 \mu \mathrm{g} / \mathrm{ml} \mathrm{L}$-ascorbic acid and $10 \mathrm{mM} \beta$-glycerophosphate, which were all obtained from Sigma-Aldrich; Merck Millipore (Darmstadt, Germany). Following $24 \mathrm{~h}$ pre-incubation and at 2,4 and 7 days following infection, supernatants were transferred to 1.5-ml Eppendorf reaction tubes (Eppendorf, Hamburg, Germany). Digestion was achieved using $65 \%$ concentrated nitric acid $(1.0 \% \mathrm{v} / \mathrm{v}$; Sigma-Aldrich, Merck Millipore). The concentration of magnesium ions released from magnesium coatings was quantified using atomic absorption spectrometry ZEEnit 650P in combination with a graphite tube atomizer (all from Analytik Jena AG, Jena, Germany). The analysis process started with evaporation steps at 80,90 and $110^{\circ} \mathrm{C}$. This was followed by a pyrolysis phase at $900^{\circ} \mathrm{C}$ in the platform tube, heated to $2,000^{\circ} \mathrm{C}$ to evaporate and transform compact fragments into atoms. This step involved elemental analysis using a resonating cathode lamp and a magnesium cathode at a wavelength of $202.6 \mathrm{~nm}$. Following 0, 2 and 7 days, the $\mathrm{pH}$ value of the supernatants was determined using an InoLab ${ }^{\circledR} \mathrm{pH} 720 \mathrm{pH}$ meter (WTW GmbH, Weilheim, Germany).

Isolation and cultivation of hOBs. The isolation of hOBs was conducted according to the protocol previously described by Jonitz et al (18). The osteoblasts were obtained from the cancellous femoral head of 6 patients that underwent total hip replacement surgery ( 3 male donors, mean age, $72 \pm 8.6$ years; 3 female donors, mean age, $78 \pm 3.8$ years) at the Department of Orthopaedics University Medicine Rostock (Rostock, Germany). The present study was approved by The Local Ethics Committee of Rostock (Rostock, Germany; registration number, A2010-10) and written informed consent was obtained from each patient.

Co-culture of hOBs and S. epidermidis. The procedures used to co-culture hOBs and S.epidermidis were the same as those described by Zaatreh et al (16). hOBs $(25,000$ cells/ml) at passage three were transferred to a 24-well-plate (Sarstedt, Nümbrecht, Germany) and cultured on the Ti6Al4V sample discs in MEM with adjunctions for $24 \mathrm{~h}$. Similarly, hOBs and S. epidermidis were cultured on Ti6Al4V sample discs coated with magnesium for $24 \mathrm{~h}$. A prepared overnight culture of S.epidermidis (RP62A; ref. no. 35984; American Type Culture Collection, Manassas, VA, USA) was subsequently used for mono-species infections with a multiplicity of infection of 0.04 (25,000 osteoblasts/ml, 1,000 colony forming units/ml). Co-cultures were incubated for a period of 7 days under standard aerobic conditions $\left(37^{\circ} \mathrm{C}\right.$ and $\left.5 \% \mathrm{CO}_{2}\right)$. At 2 and 4 days following infection the medium was replenished.

Determination of $h O B$ viability. hOBs that adhered to the discs were washed with $1 \mathrm{X}$ phosphate-buffered saline (PBS; EMD Millipore), followed by the addition of $200 \mu 1$ trypsin/EDTA (1X; Gibco; Thermo Fisher Scientific, Inc.) for 3 min under aerobic conditions $\left(37^{\circ} \mathrm{C}\right.$ and $\left.5 \% \mathrm{CO}_{2}\right)$. The hOBs were then mechanically relocated from the discs with a pipette tip (Eppendorf). The solution with the osteoblasts was transferred to a $1.5 \mathrm{ml}$ Eppendorf reaction tube (Eppendorf) centrifuged at $169 \times \mathrm{g}$ for $4 \mathrm{~min}$ at $4^{\circ} \mathrm{C}$, and washed with 1X PBS. Quantification of the number of viable primary osteoblasts on the test samples was determined by trypan blue staining (Sigma-Aldrich; Merck Millipore). Trypan blue enters the damaged membranes of dead cells, leaving viable cells unstained. The viable cells were counted using a Thoma cell counting chamber, obtained from Paul Marienfeld $\mathrm{GmbH}$ \& Co. KG (Lauda-Königshofen, Germany) according to manufacturer's protocol (19) and using an Olympus CKX41SF optical light microscope, (Olympus Soft Imaging Solutions $\mathrm{GmbH}$, Hamburg, Germany). Measurements were performed at 2 and 7 days of co-culture.

Quantification of biofilm-bound S. epidermidis. The test samples were transferred to glass test tubes (Greiner Bio-One International $\mathrm{GmbH}$, Kremsmünster, Austria) containing $1 \mathrm{ml}$ PBS (1X). S. epidermidis were removed by ultrasonic treatment with device settings at $100 \%$ for 4 min (BANDELIN BactoSonic, GmbH \& Co. KG, Berlin, Germany) at days 2 and 7 of co-culture. The solution in the glass test tube was diluted in 1X PBS and plated onto tryptic soy broth (TSB)-agar 
plates (Thermo Fisher Scientific, Inc.). Following $24 \mathrm{~h}$ incubation at $37^{\circ} \mathrm{C}$ and $5 \% \mathrm{CO}_{2}$, the number of colony forming units were quantified.

Quantification of planktonic S. epidermidis. The co-culture supernatants containing planktonic $S$. epidermidis were collected in $15 \mathrm{ml}$ centrifuge tubes (Greiner Bio-One International AG, Kremsmünster, Austria) with $1 \mathrm{ml}$ PBS (1X), and centrifuged at $3,345 \times \mathrm{g}$ for $10 \mathrm{~min}$ at $4^{\circ} \mathrm{C}$ on day 2 and 7 of co-culture. The number of planktonic bacteria were quantified by serial dilution in 1X PBS and then counting the number of colony forming units on TSB-agar plates (Thermo Fisher Scientific, Inc.) following $24 \mathrm{~h}$ incubation at $37^{\circ} \mathrm{C}$ and $5 \% \mathrm{CO}_{2}$.

Scanning electron microscopy (SEM). Test samples were fixed in a $2.5 \%$ glutaraldehyde solution at $4^{\circ} \mathrm{C}$ for $24 \mathrm{~h}$. Specimens were subsequently washed with $0.1 \mathrm{M}$ sodium acetate buffer, and dried using a graded ethanol series (5 min in 30\% ethanol, $5 \mathrm{~min}$ in $50 \%$ ethanol, $10 \mathrm{~min}$ in $70 \%$ ethanol, $15 \mathrm{~min}$ in $90 \%$ ethanol and twice for $10 \mathrm{~min}$ in $100 \%$ ethanol). The samples were then dried by critical point drying with $\mathrm{CO}_{2}$ (Critical Point Dryer, Emitech Ltd., Ashford, UK), sputter-coated with gold, and examined with a scanning electron microscope (Zeiss DSM 960A; Zeiss GmbH, Jena, Germany).

Statistical analysis. Data are expressed as the mean \pm standard error for three independent experiments. Statistical significance was evaluated with an unpaired two-tailed t-test using SPSS software (version, 20.0; IBM SPSS, Armonk, NY, USA). $\mathrm{P}<0.05$ was considered to indicate a statistically significant difference.

\section{Results}

Magnesium ion release of the test samples and $\mathrm{pH}$-values of the media. Ion release data were examined in order to characterize the strength of the corrosion process as it progresses over the 7 days. Fig. 1 shows the release of magnesium ions in the medium following one day of pre-incubation and at 2, 4 and 7 days following infection of the co-culture and the test sample alone. The magnesium-coated titanium alloy released $24 \mathrm{mM} /$ day magnesium ions during pre-incubation and $11 \mathrm{mM} /$ day over the first 2 days, decreasing to $3 \mathrm{mM} /$ day during days 4-7. The magnesium-coated surface in the co-culture with osteoblasts and bacteria released $24 \mathrm{mM} /$ day during pre-incubation and $8 \mathrm{mM} /$ day over the first 2 days, which decreased to $2 \mathrm{mM} /$ day during days 4 to 7 . Media were replenished following 1 day of pre-incubation and after 2 and 4 days of co-culture. Magnesium ion release levels in the magnesium-coated samples maintained in medium without bacteria and cells were not significantly greater when compared with the hOB and S. epidermis co-culture samples. This indicates that hOB adhesion and S. epidermidis infection did not significantly alter the release of magnesium.

Bacterial growth is influenced by $\mathrm{pH}$, therefore the $\mathrm{pH}$-values of the media were monitored. Table I indicates the $\mathrm{pH}$-values in the co-culture at $0,2,4$ and 7 days. The magnesium-coated Ti6Al4V altered the $\mathrm{pH}$-value to an alkaline environment whereas the uncoated Ti6A14V control demonstrated a slightly acidic $\mathrm{pH}$ value.
Determination of hOB viability in co-culture with S. epidermidis. The number of viable hOBs was examined by trypan blue staining (Fig. 2). A total of 25,000 cells were seeded on the two samples. The number of osteoblasts on the magnesium-coated sample was 50,000 following 2 days $(\mathrm{P}=0.01)$ and 40,000 at 7 days $(P=0.01)$. For the control, a value of 40,000 cells at day 2 and 20,000 at day 7 were observed. The number of viable osteoblasts was greater on the magnesium-coated sample when compared with the control by $\sim 10,000$ at 2 days and 20,000 at 7 days.

Evaluation of the viability of S. epidermidis in co-culture with hOBs. Measurements of bacterial colonization in the test samples revealed that a greater number of S. epidermidis colonies survived on the control TiAI6V4 surface when compared with the magnesium-coated Ti6Al4V discs (Fig. 3). The magnesium-coated sample demonstrated a significant antibacterial effect, as indicated by the significant decrease in biofilm population by three orders of magnitude following 2 days $(\mathrm{P}=0.02)$, and 4 orders of magnitude following 7 days $(\mathrm{P}=0.03$; Fig. 3). The effect was approximately 1 order of magnitude lower for the planktonic population. Complete results of the measurements for S. epidermidis are presented in Fig. 3.

SEM imaging. SEM was performed to qualitatively assess the layer structure and morphology of osteoblasts, as well as the growth patterns and distribution of bacteria on the test specimen. The SEM images shown in Fig. 4 confirm the results of the quantitative measurements of the biofilm-bound bacteria. S. epidermidis colonization was observed to be lower on the magnesium-coated surface when compared with the control on both days 2 and 7 (Fig. 4). The bacteria form biofilm-like structures on the human primary osteoblasts on the two surfaces.

\section{Discussion}

The aim of the present study was to investigate the antimicrobial properties of a rapidly corroding magnesium coating on titanium against S. epidermidis, as well as its effects on hOBs, in a co-culture model. Measurements of hOB and S. epidermidis populations revealed that hOB growth was improved and S. epidermidis growth was inhibited; these alterations differed by several orders of magnitude when compared with the uncoated titanium control.

In previously conducted monoculture experiments, the number of hOBs that colonized the magnesium-coated surface increased to $\sim 80 \%$ when compared with the uncoated titanium control, due to the slightly less favourable growth conditions on the corroding surface (14). However, in the present co-culture experiments, hOB growth on the magnesium-coated samples increased to $\sim 125 \%$ following 2 days and $200 \%$ following 7 days. The antibacterial surface significantly reduced the negative influence of $S$. epidermidis on the hOB. This result was achieved despite the presence of the magnesium coating, which demonstrates a negative effect on the growth potential of the hOBs. The negative influence of S. epidermidis on hOB may be due to competition for nutrients and pathogenicity (20). The uninhibited negative effects was observed on the control sample, where hOB numbers were markedly reduced following 
Table I. pH-values for Ti6A14V and Mg-coated Ti6A14V test samples in co-culture with hOB and S. epidermidis for 0, 2, 4 and 7 days.

\begin{tabular}{lcccr}
\hline Co-culture of hOB and S. epidermidis & Day 0 & Day 2 & Day 4 & Day 7 \\
\hline MEM+10\% FCS & $7.94 \pm 0.07$ & $8.03 \pm 0.15$ & $8.01 \pm 0.07$ & $8.05 \pm 0.03$ \\
Ti6Al4V & $7.88 \pm 0.06$ & $7.83 \pm 0.08$ & $7.81 \pm 0.03$ & $7.64 \pm 0.04$ \\
Mg-coating & $8.82 \pm 0.08$ & $8.56 \pm 0.08$ & $8.19 \pm 0.01$ & $8.12 \pm 0.04$ \\
\hline
\end{tabular}

Data are expressed as the mean \pm standard deviation $(n=3)$. MEM, Modified Eagle's osteogenic cell culture medium; FCS, foetal calf serum; hOBs, human osteoblasts; S. epidermidis, Staphylococcus epidermidis; Mg, magnesium.

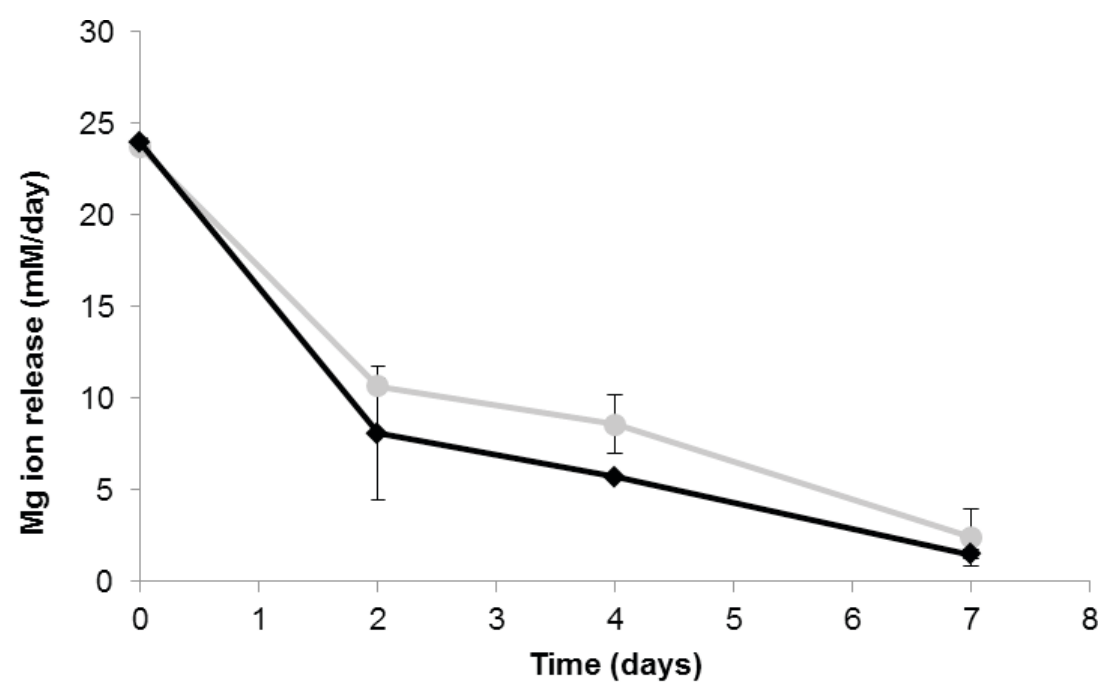

- Mg coating $\rightarrow$ Mg coating + hOB $+S$. epidermidis

Figure 1. Magnesium ion release in the co-culture and test sample-only systems. Mg ion release was measured in the medium following 1 day of pre-incubation and at 2, 4 and 7 days for test sample in medium alone and test sample co-cultured with hOBs and S. epidermidis, as determined by atomic absorption spectrometry. The release declines from $\sim 24 \mathrm{mM} /$ day on day 1 of exposure to the medium, to $2-3 \mathrm{mM} /$ day on day 7 in the co-culture and test sample-only systems. Data are expressed as the mean \pm standard error $(\mathrm{n}=3)$. S. epidermidis, Staphylococcus epidermidis; hOBs, human osteoblasts; $\mathrm{Mg}$, magnesium.

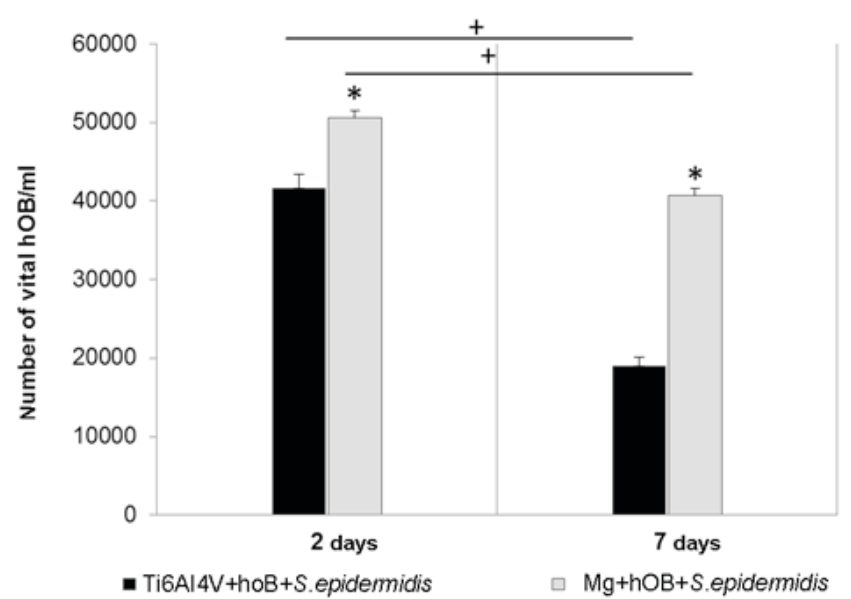

Figure 2. Viability of human hOBs in the co-culture on the test samples for 2 and 7 days. The number of osteoblasts on the titanium alloy sample was decreased by $\sim 20,000 \mathrm{hOB} / \mathrm{ml}$ at day 7 compared to day 2 , whereas a reduction of $10,000 \mathrm{hOB} / \mathrm{ml}$ was observed on the $\mathrm{Mg}$-coated samples. Data are expressed as the mean \pm standard error of the mean $(n=3)$. ${ }^{*} \mathrm{P}<0.05$ vs. Ti6Al4V + hOB + S. epidermidis (same day). ${ }^{+} \mathrm{P}<0.05$ vs. day 2 (same material). S. epidermidis, Staphylococcus epidermidis; hOBs, human osteoblasts; Mg, magnesium.
7 days when compared with 2 days (a decrease of 20,000 from 40,000).

The increased growth of the hOBs on magnesium-coated samples, and the observed antibacterial effect, may be explained by the following factors: Prevention of bacterial adherence due to the corrosive dissolution process, osmotic stress in the strongest phase of initial corrosion, the microstructure of the surface, an unfavourable increase in $\mathrm{pH}$-value for the bacteria or the inhibitory effects of magnesium ions on the bacteria (21-28). These effects are most prominent in the local environment close to the surface. The effects of dissolution and osmotic pressure decline over 2 days, however the $\mathrm{pH}$ shift may also have a negative effect on S. epidermidis growth over the 7-day period. The impact on the planktonic population may be less significant due to reduced exposure to these factors $(13,29)$.

The antibacterial effects observed in the monoculture experiments were reproduced in the co-culture setup, however, they were observed to be one order of magnitude less prominent (14). These observations are in agreement with a prior comparison of a monoculture to co-culture experiment conducted by Zaatreh et al (16). Putative reasons for the 


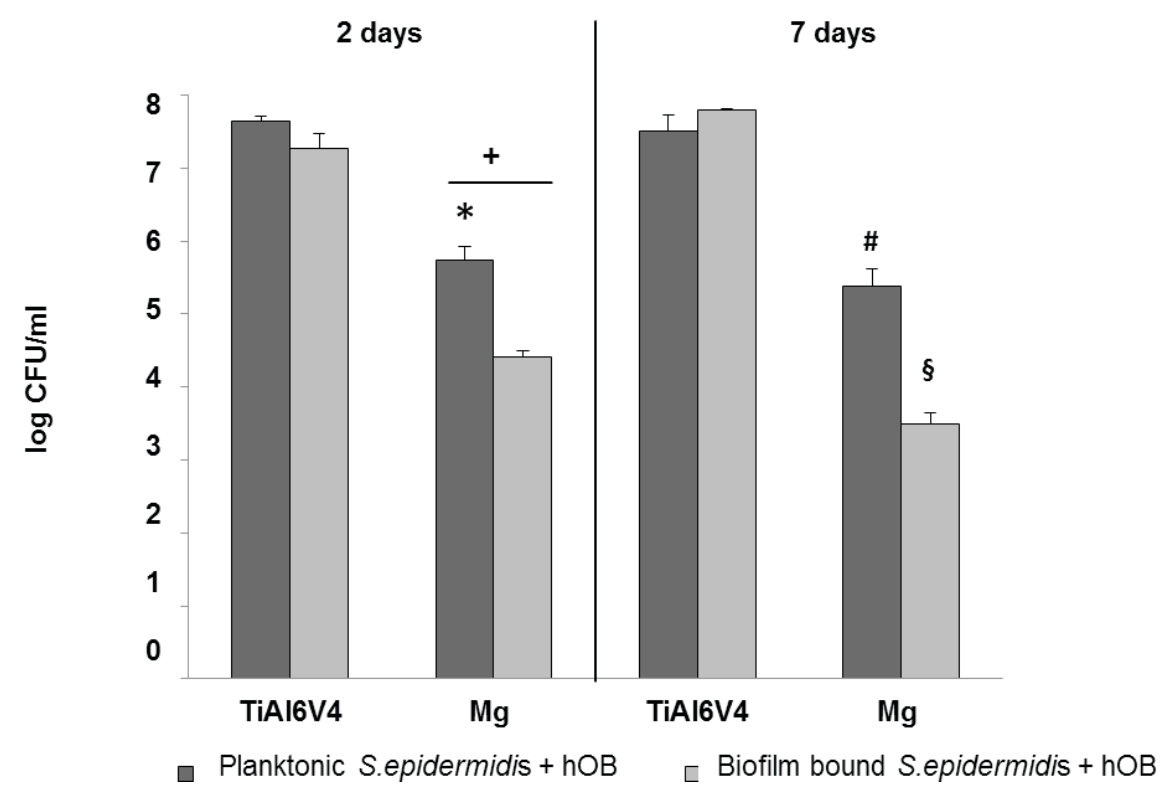

Figure 3. Quantification of viable biofilm-bound and planktonic S. epidermidis in co-culture with hOB cells on the test samples following 2 and 7 days. The number of viable biofilm-bound S. epidermidis on the Mg-coated specimen was significantly decreased when compared with the titanium control over a period of 7 days. Data are expressed as the mean \pm standard error of the mean $(\mathrm{n}=3)$. ${ }^{*} \mathrm{P}<0.05 \mathrm{Mg}$ vs. titanium alloy control, day 2 , planktonic. ${ }^{+} \mathrm{P}<0.05$ biofilm vs. planktonic, day 2, on Mg. ${ }^{\prime \prime} \mathrm{P}<0.05 \mathrm{Mg}$ vs. titanium alloy control, day 7, planktonic. ${ }^{\circledR} \mathrm{P}<0.05 \mathrm{Mg}$ vs. titanium alloy control, day 7 , biofilm. S. epidermidis, Staphylococcus epidermidis; hOBs, human osteoblasts; $\mathrm{Mg}$, magnesium; $\mathrm{CFU}$, colony forming units.

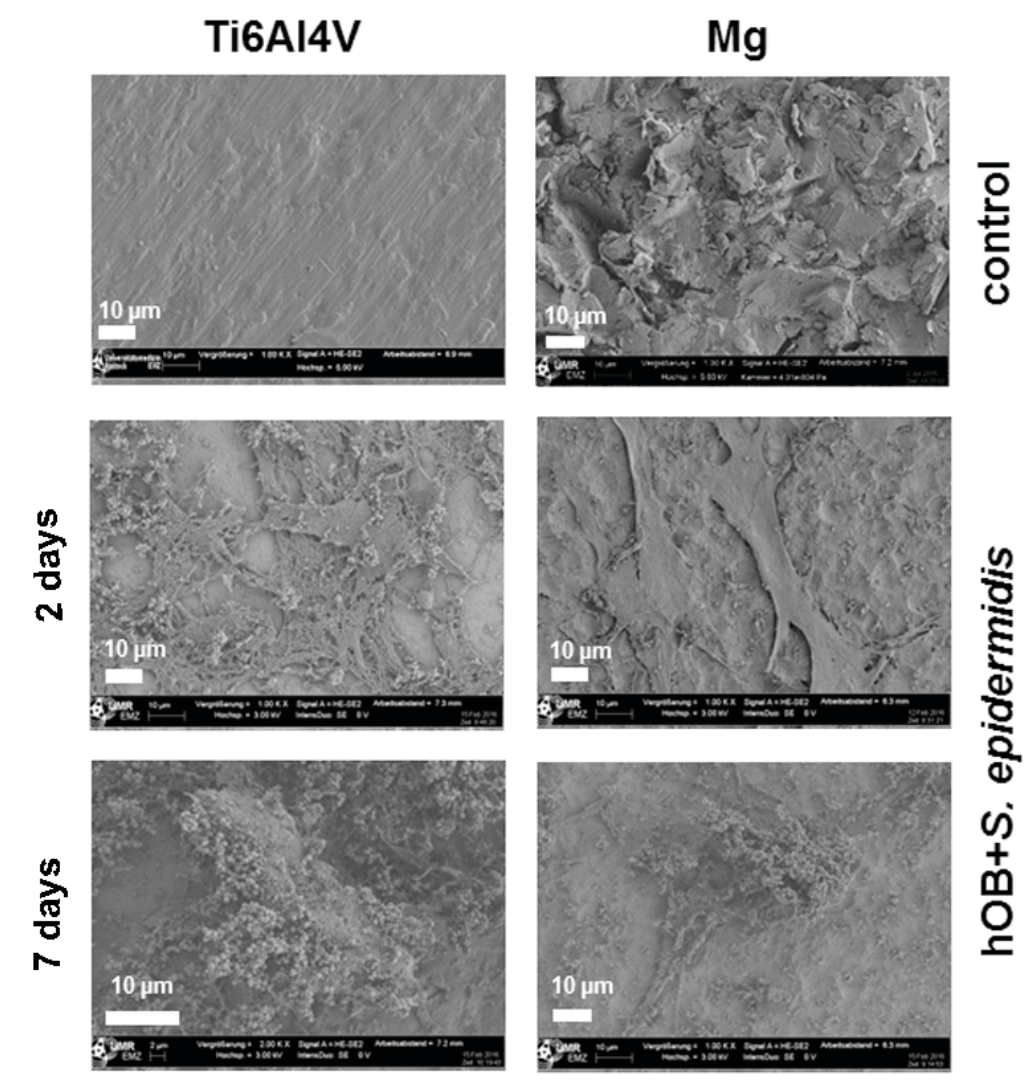

Figure 4. Scanning electron microscopy images of S. epidermidis in co-culture with hOB on the titanium-only test sample and the Mg-coated test sample for 2 and 7 days. A decreased density of S. epidermidis colonization was observed on the Mg-coated surface when compared with the titanium-only test sample (magnification, $\mathrm{x} 1,000$ or $\mathrm{x} 2,000$ ). Control images are test samples in medium only. S. epidermidis, Staphylococcus epidermidis; hOBs, human osteoblasts; $\mathrm{Mg}$, magnesium.

diminished effect may include the favourable environment that the hOBs present for S. epidermidis and the protection that they may provide from antibacterial effects of the surface.
The planktonic population of S. epidermidis depends on the biofilm population, as this is where most of the bacterial reproduction occurs (30). 
In previous studies, the authors demonstrated that monocultures may capture only a limited number of interactions among human cells, bacteria and the tested surface $(14,16)$. Unpredicted behaviour may arise from the complex interaction among human cells, bacteria and implant surfaces. Therefore, the results of co-culture experiments are regarded as more robust $(31,32)$. In the present study, the co-culture model captured the apparent protective effects of the hOB cells, whereby this layer of cells protects bacteria from the adverse effects of the surface. The antibacterial effects were diminished in the co-culture system, however a significant antibacterial effect persisted (three orders of magnitude following 7 days).

The investigation of different experimental parameters must be limited. Future work will aim to test different growth media, a greater number of bacterial strains and species, as well as different osteoblast cell lines. In addition, the length of time for infection to occur, and the ratio of initial hOB and bacterial numbers will be explored. The results of the present study provide significant observations with promising implications for implant material research.

In conclusion, novel implant materials are in high demand due to the severity of IAI, which may be life-threatening for patients with lengthy and demanding treatment procedures and may result in a marked decrease in quality of life. It has been previously demonstrated that titanium coated with a thin, rapidly corroding layer of magnesium may be a suitable candidate material, based on monoculture studies involving primary hOBs and $S$. epidermidis. The present study applied a more rigorous test system with hOBs and $S$.epidermidis in co-culture on the magnesium-coated titanium surface. The favourable qualities of this surface as an antibacterial and biocompatible material were verified. These results provide evidence for progression towards future animal model testing.

\section{Acknowledgements}

The authors would like to thank Professor Andreas Podbielski (Institute of Medical Microbiology, Virology and Hygiene, University Medicine Rostock) for his help in performing the tests. In addition, the authors would like to thank Dr Marcus Frank, Mr. Wolfgang Labs, Mr. Gerhard Fulda (Electron Microscopy Centre, University Medicine Rostock) Mr. Philipp Pisowocki (Department of Orthopaedics, University Medicine Rostock) and Mrs. Regina Lange (Institute for Electronic Appliances and Circuits, University Medicine Rostock) for their help with SEM. Furthermore, a special thanks to Professor Regine Willumeit-Römer (Institute of Materials Research, Division of Metallic Biomaterials, Helmholtz-Zentrum Geesthacht) for her support and expertise in the field of magnesium as a biomaterial. This study was financially supported by the Helmholtz Virtual Institute MetBioMat (grant no. VH-VI-523) titled 'In vivo studies of biodegradable magnesium-based implant materials'.

\section{References}

1. Fallpauschalenbezogene Krankenhausstatistik (DRG-Statistik): Operationen und Prozeduren der vollstationären Patientinnen und Patienten der Krankenhäuser (4-Steller). Statistisches Bundesamt (Destatis), Wiesbaden, 2015.
2. Darouiche RO: Treatment of infections associated with surgical implants. N Engl J Med 350: 1422-1429, 2004.

3. Walter RP and Blake SM: Re: Two-stage exchange for infected resurfacing arthroplasty use of a novel cement spacer technique. J Arthroplasty 26: 979, 2011.

4. Harris LG and Richards RG: Staphylococci and implant surfaces: A review. Injury 37 (Suppl 2): S3-S14, 2006.

5. Casey AL, Lambert PA and Elliott TSJ: Staphylococci. Int J Antimicrob Agents 29 (Suppl 3): S23-S32, 2007.

6. Arciola CR, Campoccia D, Speziale P, Montanaro L and Costerton JW: Biofilm formation in Staphylococcus implant infections. A review of molecular mechanisms and implications for biofilm-resistant materials. Biomaterials 33: 5967-5982, 2012

7. Wilde AH and Ruth JT: Two-stage reimplantation in infected total knee arthroplasty. Clin Orthop: 23-35, 1988.

8. Vorndran E, Spohn N, Nies B, Rössler S, Storch S and Gbureck U: Mechanical properties and drug release behavior of bioactivated PMMA cements. J Biomater Appl 26: 581-594, 2012.

9. Uchiyama K, Takahira N, Fukushima K, Moriya M, Yamamoto T, Minegishi Y, Sakai R, Itoman M and Takaso M: Two-stage revision total hip arthroplasty for periprosthetic infections using antibiotic-impregnated cement spacers of various types and materials. ScientificWorldJournal 2013: 147248, 2013.

10. Connaughton A, Childs A, Dylewski S and Sabesan VJ: Biofilm disrupting technology for orthopedic implants: What's on the Horizon? Front Med (Lausanne) 1: 22, 2014.

11. Staiger MP, Pietak AM, Huadmai J and Dias G: Magnesium and its alloys as orthopedic biomaterials: A review. Biomaterials 27: $1728-1734,2006$

12. Luthringer BJ, Feyerabend $F$ and Willumeit-Römer R: Magnesium-based implants: A mini-review. Magnes Res 27: 142-154, 2014.

13. Robinson DA, Griffith RW, Shechtman D, Evans RB and Conzemius MG: In vitro antibacterial properties of magnesium metal against Escherichia coli, Pseudomonas aeruginosa and Staphylococcus aureus. Acta Biomater 6: 1869-1877, 2010.

14. Zaatreh S, Haffner D, Pasold J, Kreikemeyer B, Mittelmeier W, Podbielski A, Quandt E and Bader R: Track A. Biomaterials and biocompatibility 1. Biomed Tech (Berl) 60 (Suppl 1): S1-S30, 2015.

15. Cytotoxicity test according to ISO 10993-5. CYTOX, 1999.

16. Zaatreh S, Wegner K, Strauß M, Pasold J, Mittelmeier W, Podbielski A, Kreikemeyer B and Bader R: Co-culture of S. epidermidis and human osteoblasts on implant surfaces: An advanced in vitro model for implant-associated infections. PloS One 11: e0151534, 2016.

17. Schlüter K, Zamponi C, Piorra A and Quandt E: Comparison of the corrosion behaviour of bulk and thin film magnesium alloys. Corros Sci 52: 3973-3977, 2010.

18. Jonitz A, Lochner K, Lindner T, Hansmann D, Marrot A and Bader R: Oxygen consumption, acidification and migration capacity of human primary osteoblasts within a three-dimensional tantalum scaffold. J Mater Sci Mater Med 22: 2089-2095, 2011.

19. Anleitungen-Marienfeld-Superior: [Internet]. [cited 3 Nov 2016]. Available: http://www.marienfeld-superior.com/index.php/anleitungen.html

20. Von Eiff C, Peters G and Heilmann C: Pathogenesis of infections due to coagulasenegative staphylococci. Lancet Infect Dis 2: $677-685,2002$

21. Wang HX, Guan SK, Wang X, Ren CX and Wang LG: In vitro degradation and mechanical integrity of $\mathrm{Mg}-\mathrm{Zn}-\mathrm{Ca}$ alloy coated with Ca-deficient hydroxyapatite by the pulse electrodeposition process. Acta Biomater 6: 1743-1748, 2010.

22. Kirkland NT, Lespagnol J, Birbilis N and Staiger MP: A survey of bio-corrosion rates of magnesium alloys. Corros Sci 52: 287-291, 2010.

23. Fischer J, Prosenc MH, Wolff M, Hort N, Willumeit R and Feyerabend F: Interference of magnesium corrosion with tetrazolium-based cytotoxicity assays. Acta Biomater 6: 1813-1823, 2010.

24. Fischer J, Pröfrock D, Hort N, Willumeit R and Feyerabend F: Reprint of: Improved cytotoxicity testing of magnesium materials. Mater Sci Eng B 176: 1773-1777, 2011.

25. Schlüter K, Zamponi C, Hapke J, Hort N, Kainer KU and Quandt E: Mechanical properties and corrosion behaviour of freestanding, precipitate-free magnesium WE43 thin films. Int J Mater Res 104: 286-292, 2013.

26. Schlüter K, Shi Z, Zamponi C, Cao F, Quandt E and Atrens A: Corrosion performance and mechanical properties of sputter-deposited MgY and MgGd alloys. Corros Sci 78: 43-54, 2014. 
27. Martinez Sanchez AH, Luthringer BJC, Feyerabend F and Willumeit $\mathrm{R}: \mathrm{Mg}$ and $\mathrm{Mg}$ alloys: How comparable are in vitro and in vivo corrosion rates? A review. Acta Biomater 13: 16-31, 2015.

28. Willumeit R, Feyerabend F and Huber N: Magnesium degradation as determined by artificial neural networks. Acta Biomater 9: 8722-8729, 2013.

29. Charyeva O, Neilands J, Svensäter G and Wennerberg A: Bacterial biofilm formation on resorbing magnesium implants. Open J Med Microbiol 5: 1-11, 2015.

30. Gasol JM and Del Giorgio PA: Using flow cytometry for counting natural planktonic bacteria and understanding the structure of planktonic bacterial communities. Sci Mar 64: 197-224, 2000
31. Lee JH, Wang H, Kaplan JB and Lee WY: Microfluidic approach to create three-dimensional tissue models for biofilm-related infection of orthopaedic implants. Tissue Eng Part C Methods 17: 39-48, 2011.

32. Kaji H, Camci-Unal G, Langer R and Khademhosseini A: Engineering systems for the generation of patterned co-cultures for controlling cell-cell interactions. Biochim Biophys Acta 1810: 239-250, 2011. 